\title{
Python Predictive Analysis for Bug Detection
}

\author{
Zhaogui $\mathrm{Xu}^{*}$, Peng Liu ${ }^{\dagger}$, Xiangyu Zhang ${ }^{\ddagger}$, and Baowen $\mathrm{Xu}^{* \ddagger}$ \\ * State Key Laboratory of Novel Software Technology † Department of Computer Science \\ * Nanjing University, China \\ ${ }^{\dagger}$ Purdue University, USA \\ zgxu@smail.nju.edu.cn \{peng74,xyzhang\}@cs.purdue.edu bwxu@nju.edu.cn
}

\begin{abstract}
Python is a popular dynamic language that allows quick software development. However, Python program analysis engines are largely lacking. In this paper, we present a Python predictive analysis. It first collects the trace of an execution, and then encodes the trace and unexecuted branches to symbolic constraints. Symbolic variables are introduced to denote input values, their dynamic types, and attribute sets, to reason about their variations. Solving the constraints identifies bugs and their triggering inputs. Our evaluation shows that the technique is highly effective in analyzing real-world complex programs with a lot of dynamic features and external library calls, due to its sophisticated encoding design based on traces. It identifies 46 bugs from 11 real-world projects, with 16 new bugs. All reported bugs are true positives.
\end{abstract}

\section{CCS Concepts}

-Software and its engineering $\rightarrow$ Software testing and debugging;

\section{Keywords}

Python; Dynamic Language; Debugging; Predictive Analysis

\section{INTRODUCTION}

Python is becoming one of the most widely used programming languages due to its flexibility, usability, and strong library support for quick development. According to IEEE Spectrum [1], Python is one of the four most popular programming languages in 2015.

Due to the prevalence of Python, analyzing Python programs becomes as important as analyzing programs in traditional languages such as Java and C. However, analyzing Python programs is much more difficult due to the following

\footnotetext{
$\ddagger$ Corresponding authors.
}

Permission to make digital or hard copies of all or part of this work for personal or classroom use is granted without fee provided that copies are not made or distributed for profit or commercial advantage and that copies bear this notice and the full citation on the first page. Copyrights for components of this work owned by others than ACM must be honored. Abstracting with credit is permitted. To copy otherwise, or republish, to post on servers or to redistribute to lists, requires prior specific permission and/or a fee. Request permissions from Permissions@acm.org.

FSE'16, November 13-18, 2016, Seattle, WA, USA

(C) 2016 ACM. 978-1-4503-4218-6/16/11...\$15.00

http://dx.doi.org/10.1145/2950290.2950357 reasons. First of all, Python is dynamically typed. Variables have no static type declarations and may hold values of different types at runtime. The signatures of Python functions also have no type declarations for the parameters, which allows the functions to accept any types of parameters. Secondly, everything in Python is an object. Particularly, functions, classes and other kinds of objects are all first-class objects that can be created and passed anywhere. Thirdly, object attributes can be dynamically accessed and updated through computed names (e.g., through reflection functions getattr() and setattr( )). Fourthly, collections are pervasively used in Python. They are usually heterogeneous so that they may hold any types of values. Finally, external function calls are pervasive in Python programs.

Existing analysis for Python and other dynamic languages can be classified to three kinds. Static analysis [25, 23, 2, 3] (e.g., abstract interpretation and type inference) analyze program statically. They have limited effectiveness due to the dynamic features in Python. Dynamic analyses $[11,13]$ focus on the observed executions, and hence cannot analyze unexecuted paths. Symbolic and concolic execution engines $[15,17,18,19]$ are developed for dynamic languages such as Python, Javascript, and Ruby. These engines explore individual paths in a program. Many of them do not support exploring other aspects of the state space such as type variations and attribute set variations that are very common in dynamic languages, due to state explosion and difficulties in explicitly encoding these variations.

In this paper, we develop a predictive analysis engine for Python programs. Predictive analysis $[4,5,6,7]$ is a kind of symbolic analysis driven by concrete executions. It first collects the execution trace of a passing run. It then symbolically encodes the trace, and relaxes the parts that the analyst wants to reason about by introducing symbolic variables to denote possible variations (e.g., schedules in a multi-threaded program execution). Solving the constraints is equivalent to exploring the executions that have small differences from the traced execution to find bugs. We call these the neighboring executions of the observed execution.

Our contributions are summarized as follows.

- We develop the first predictive analysis engine for Python programs. It explores neighboring executions of an observed execution by relaxing the inputs, including their types, attribute sets, and values. Different from most predictive analysis that encodes schedule variations and prohibits path changes, it encodes input variations and handles the resulting state changes 
including path changes. Like other predictive analysis, ours is sound, never reporting false positives.

- We design a novel encoding scheme for Python programs and execution traces. It focuses on handling dynamic features by introducing symbolic variables to represent dynamic types and attributes, and explicitly reasoning about their changes and correlations.

- As part of the engine, we develop a tracing infrastructure for Python programs. It features tracing not only the traditional control flow and data flow information, but also the implicit dynamic properties of executed Python statements such as object attributes.

- We build a prototype and evaluate it on 11 real-world Python programs. We have identified totally 46 real bugs, and 16 of them were never found before. The analysis time is reasonable.

The Essence of Our Technique. Symbolic/concolic analysis explore program state space leveraging concrete and symbolic reasonings. The entire program state space can be divided into three parts: (1) sub-space that is not explored (e.g., the concretized values in concolic execution); (2) sub-space implicitly explored by the solver (e.g., schedule variations in traditional predictive analysis); and (3) sub-space explicitly explored by the engine not the solver (e.g., path exploration in symbolic execution). Different symbolic/concolic analysis differ in the distribution of the three sub-spaces. For example, traditional symbolic/concolic execution engines (e.g., $[18,15,16]$ ), including those for dynamic languages, explicitly explore individual program paths. Along each path, they leverage the solver to implicitly explore variable values along the path. Explicit exploration substantially simplifies constraint encoding as only one path is encoded at a time. State variations caused by path variations do not need to be encoded. However, identifying sophisticated exploration strategies and symbolic state checkpointing/backtracking are challenging. Implicit exploration can achieve efficiency leveraging the built-in demanddriven nature of constraint solving in modern solvers. Its difficulties lie in the complexity of constraint encoding.

Compared to existing symbolic/concolic execution, our technique has the following unique features that make it suitable for Python. (1) It encodes values, types, attribute sets, paths, and their correlations so that the solver can be leveraged to perform efficient implicit exploration, whereas most existing techniques explicitly explore paths and do not reason about type or attribute variations that are critical for dynamic languages. (2) The user provides initial (passing) executions to direct our system to the functionalities/components in which they want to find bugs. For each initial execution, our technique encodes its neighboring state space including paths not executed in the initial run. In other words, one request (to the solver) allows implicit exploration of all the executions in the neighborhood. This completely avoids the highly demanding explicit path exploration. (3) Modeling for the large number of external functions can be substantially mitigated by trace preprocessing (Section 4.3).

\section{MOTIVATING EXAMPLE}

In this section, we use a simplified problematic Python program to motivate our approach.

Fig. 1 shows the code snippet simplified from the very popular Python HTTP library requests. The test driver function test_post() invokes the function post at line 276

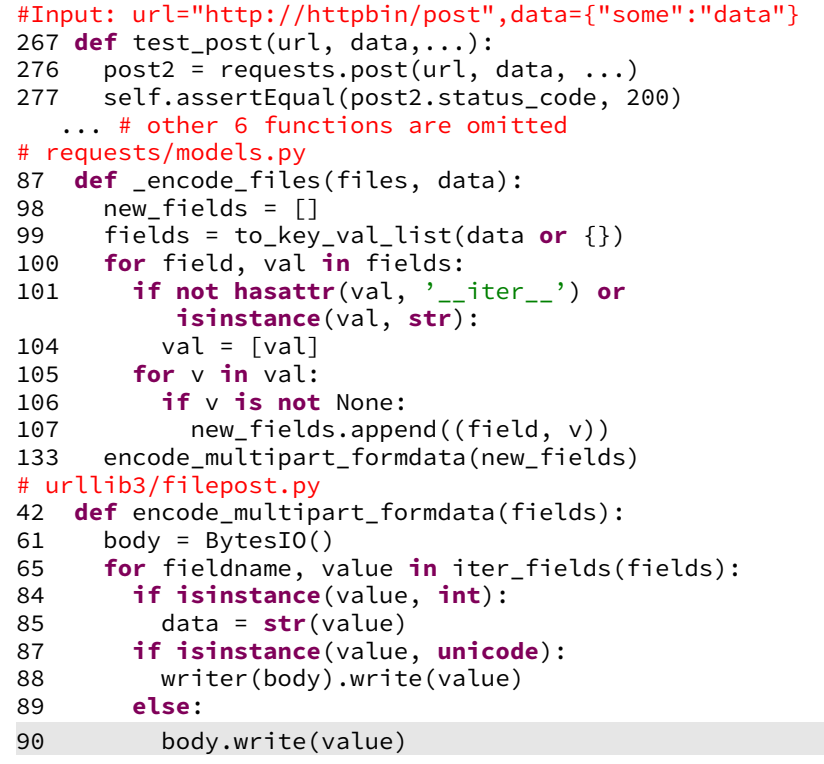

Figure 1: A Motivating Example. (_encode_files() is an internal function called during execution whose argument data comes from the test input. Unrelated code is omitted for readability.)

and checks its return value at line 277 . The input parameter data can be a dictionary.

Suppose the input parameter data is \{"some": "data"\}. The function call post () at line 276 invokes the function _encode_files() (lines 87-133) transitively through a chain that consists of six function calls (omitted for simplicity). Line 99 converts the dictionary data into a list of pairs, where each pair represents an entry of the dictionary. The resultant list fields is [("some", data")]. Line 100 iterates over the list and unpacks each pair to field and val. For the pair ("some", "data"), the unpacked field and val are "some" and "data", respectively. Val is wrapped as a list (line 104) if it is not iterable (i.e., without the attribute "_iter_") or is of the str type (line 101). Here, val is wrapped as a list since it is of str type. Lines 105-107 then iterate over val, form a pair with field and store the pair into the list new_fields. In this example, new_fields is [("some", "data")]. New_fields is then passed to the function encode_multipart_formdata() as the parameter fields. Lines $42-90$ iterate over fields and check the value in each pair. If it is not of the int (line 84) or unicode (line 87) type, e.g., it is the string "data" in our example, it is written to the body (line 90).

The defect is at line 90 . The method body.write() requires the parameter to implement the buffer interface. For example, the str type implements the interface while the float type does not. If body.write() takes a float value (e.g., 0.0) as parameter, it will crash. Our approach is able to compute the new input data, \{"some": $\odot . \odot\}$, to trigger such a crash.

The example illustrates a number of the inherent challenges in analyzing Python programs. Observe that all variables are dynamically typed such as value at line 90 . Function parameters can also accept different types. Type errors are only captured by crashes. This example is difficult for static analysis as it involves a lot of container related operations (e.g., the operations in _encode_files ()) which require complex heap abstraction. This example also im- 


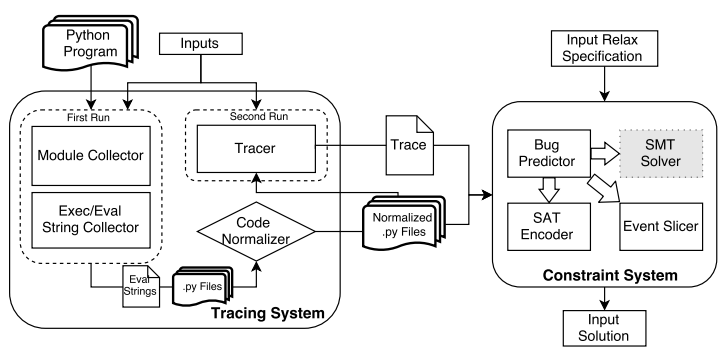

Figure 2: System Framework

poses challenges to symbolic analysis. For example, the bug manifests in a very deep call chain. The execution has a lot of invocations to external functions, which require substantial efforts to model. In fact, the traced run for the example executes more than 18000 Python statements, among them there are about 2700 container operations and 99 reflection function calls. There are also 946 external functions calls. In addition, we cannot analyze the function encode_multipart_formdata() in isolation. In fact, the function is also used in a library urllib3, where its use is not buggy since the library performs dynamic checks to ensure the inputs are of the proper types during the function calls. Our Solution. Our technique belongs to the category of predictive analysis, which starts with the trace of an existing run and predicts a new feasible run. Given the trace collected from the passing run with the input \{"some": "data"\}, our analysis first models the inputs, including their values, types, and attribute sets, as symbolic variables to denote possible input variations; then it encodes the executions possibly derived from the trace into constraints; next it encodes the bug finding assertions, e.g., the value is not of the type str or bytearray (for line 90), which implements the buffer interface; lastly it leverages a solver to compute the input that triggers any assertion violation. In this example, we compute the input data as $\{$ "some": $\odot . \odot\}$. The predicted run follows the same path as the observed run to reach line 90 and executes body.write $(\odot . \odot)$, which leads to the crash.

Our approach fully supports dynamic typing and dynamic attribute updates in Python. The readers may notice that the input contains different types/attributes in the original run and the predicted run (i.e., \{"some": "data"\} and \{"some": $\odot . \odot\}$ ), and value at line 90 has different types/attributes in the two runs. Our system introduces auxiliary type/attribute symbolic variables to track type/attribute information explicitly and supports type/attribute checks (e.g., lines 84 and 87). In contrast, most existing symbolic execution engines do not reason about type/attribute variations, and hence have difficulties finding the bug.

Our approach not only encodes the current execution path (as in many existing symbolic/concolic execution engines), but also encodes the unexecuted branches together with the path. For example, lines 85 and 88 were not executed in the original run but our technique encodes them with the original run so that the solver can explore these branches implicitly. In contrast, existing symbolic/concolic engines follow some strategy (e.g., depth-first search) to explicitly explore the unexecuted branches through many runs. Details will be discussed in section 5.2.

Besides dynamic types and attribute sets, our approach also supports other dynamic features such as reflection calls and eval (), leveraging the traces.

\section{SYSTEM FRAMEWORK}

In this section, we give an overview of the system. As shown in Fig. 2, it consists of two components: the tracing component and the constraint encoding/solving component.

The tracing component takes a Python program and a test input, and produces a trace of the execution. The tracing procedure consists of three steps. First, the program is run to collect all the involved modules and the string parameters of the eval() function invocations, which are loaded or constructed at runtime and are unknown at compile time. This step essentially collects the complete set of executed code. Second, we normalize the code (collected from the modules and the eval () parameters) by breaking the complex statements into simple statements. Finally, we run the normalized program again to collect the trace. One prominent challenge in tracing Python program execution is to handle a large number of external function calls that are implemented in other languages and have unknown sideeffects. Our tracing component also features an on-the-fly side-effect detection technique to handle external functions.

The constraint encoding/solving component takes three resources as inputs, namely, the trace, the normalized source code and the input relaxation specification ${ }^{1}$. Our system reports bugs and the corresponding inputs that trigger the bugs. The constraint system consists of four subcomponents: the bug predictor, the constraint encoder, the event slicer and the SMT solver. The bug predictor subcomponent is a controller that scans the execution trace. During the procedure, it may invoke the other three components. When it finds a satisfiable solution, meaning a bug, it will stop evaluating further. The constraint encoder mainly performs three actions: (1) relaxing the encodings of inputs according to the input specification such that the encoding describes a much larger state space than the traced execution; (2) encoding the execution trace to constraints; (3) aggressively including the unexecuted branches to the constraints whenever encountering a predicate event (in the trace). A trace slicer is used to slice events on which a specific assertion event (i.e., the slice criterion) is dependent. We provide the constraints of the slice to an SMT solver. If it is satisfiable, we find a bug and its triggering input. Otherwise, our tool does not find a violation to the assertion and moves on to the next assertion.

\section{TRACING COMPONENT}

Similar to existing methods of predictive analysis [4, 5, 8], we trace an execution based on 3-address instructions, which are more suitable for constraint encoding than bytecode instructions. Our tracing component follows three steps to collect trace. (1) It executes the program for the first time, and collects all the involved modules and string parameter values for the exec()/eval() calls. (2) With the collected modules and string values, it normalizes the source code to a simplified form with each line only having one simple operation. (3) After replacing the original modules with the normalized versions, it re-executes the program and in the meantime collects the execution trace.

\subsection{Code Normalization}

The official releases of Python have a built-in tracing API sys.settrace allowing hooking the execution environment

\footnotetext{
${ }^{1}$ We put the specification design into our technical report for reference: https://sites.google.com/site/pypredictor/
} 
before executing each source code line, through which we can perform a set of queries to the runtime environment, making tracing and gathering runtime information very convenient (e.g., querying values, types and attributes). However, the API only allows tracing at the level of source code line, which is problematic for our purpose as one source code line may contain multiple statements and a long statement may cross multiple lines. As a result, we need to normalize the source code such that each line only contains a simple operation. We perform the following three kinds of normalization.

Linearizing Nested Expressions. Nested Expressions are prevalent in Python code, which combine multiple operations together(e.g., a.b. $f()+x)$. We linearize these nested expressions into a group of simple assignments, each containing one simple expression.

Eliminating Complex Constructs. Python has very expressive syntax, so that complex semantics can be represented concisely. While it provides convenience for programmers, it creates difficulties for analysis. Our system transforms these advanced constructs to a small set of simple constructs. We list several typical transformations as follows.

\begin{tabular}{|c|c|}
\hline $\begin{array}{r}x=[f(i) \text { for } i \text { in lst } \\
\text { if } i>0]\end{array}$ & $\begin{array}{l}x=[] \\
\text { for } i \text { in lst: } \\
\text { if } i>\odot: \\
\quad \text { tmp }=f(i) \\
\quad x \text {.append }(\text { tmp })\end{array}$ \\
\hline $\begin{array}{c}x=\operatorname{reduce}(\text { lambda } a, b: a+b, \backslash \\
\text { lst) }\end{array}$ & $\begin{array}{l}\text { def } v 1(a, b): \\
v 2=a+b \\
\text { return } v 2 \\
x=\operatorname{reduce}(v 1, \text { lst })\end{array}$ \\
\hline
\end{tabular}

The first row presents a concise method to construct a list by applying a function $f()$ to the loop index $i$, with the transformed code on the right. The second row illustrates how we transform a lambda expression.

Transforming eval()/exec(). Our system also replaces eval()/exec() calls with the corresponding real code collected earlier. Consider the following example.

\begin{tabular}{|c|c|c|}
\hline $\begin{array}{l}\# a \cdot p y \\
10: \text { co }=x+"=a " \\
11: \text { exec }(c o)\end{array}$ & $\begin{array}{l}\# \text { records } \\
\langle a \# 11, c o, b=a\rangle \\
\langle a \# 11, c o, c=a\rangle\end{array}$ & $\begin{array}{l}c o=x+"=a " \\
\text { if } c o==" b=a ": \\
b=a \\
\text { elif } c o==" c=a ": \\
c=a \\
\text { else: } \\
\quad \text { exec }(c o)\end{array}$ \\
\hline
\end{tabular}

The left column shows the original code that contains an exec () call at line 11. The middle column presents the trace records of this line, in which " $b=a$ " and " $c=a$ " are the values of variable co in the two records. The right column shows the corresponding code that our system uses to replace the call. Observe that we use branches to handle the different statements induced by the different values of co. We also reserve the original exec(co) in the else branch to ensure soundness. With the transformed code, we now can trace the execution of the original exec() call.

\subsection{Trace Generation}

After replacing the involved modules with the normalized ones, we now re-execute the program to gather the execution trace. Tracing Python program execution is more challenging than tracing programs in many other languages because the execution of a Python statement may have many implicit side-effects, such as type changes and attribute set changes. All these need to be explicitly captured as many bugs are related to these implicit side-effects. Next, we first introduce some notations and then illustrate the tracing rules.

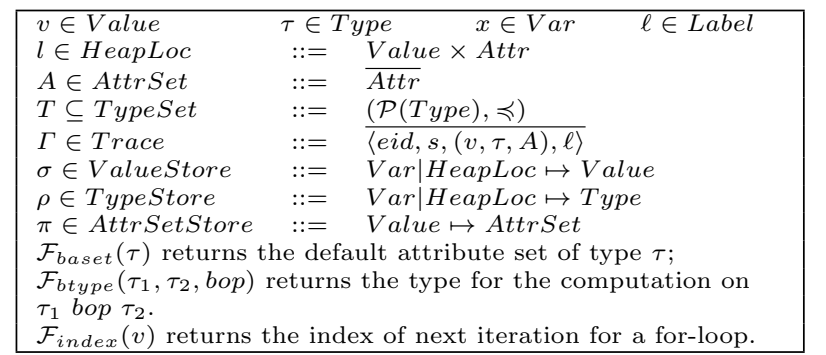

Figure 3: Definitions Related to Tracing Rules

\begin{tabular}{rl|l|}
\hline Statement $s::={ }^{\ell} v|x|(x$ op $y)$ & $\mid$ (basic assignments) \\
$y={ }^{\ell} x . f$ & $\mid$ (attr-read) \\
$y \cdot f={ }^{\ell} x \mid v$ & $\mid$ (attr-write) \\
if $(x \text { relop } y)^{\ell} s_{1}$ else $s_{2}$ & $\mid$ (cmp-predicate) \\
if isinst $(x, t)^{\ell} s_{1}$ else $s_{2}$ & $\mid$ (isinst-predicate) \\
if hasattr $(x, f)^{\ell} s_{1}$ else $s_{2}$ & $\mid$ (hasattr-predicate) \\
$y=f^{\ell}\left(a_{1}, \ldots, a_{n}\right) \mid x \cdot m^{\ell}\left(a_{1}, \ldots, a_{n}\right)$ & $\mid$ (callsite)
\end{tabular}

Figure 4: Python Statements

Fig. 3 presents the definitions related to the tracing rules. Basically, $v$ represents an arbitrary value. Note that every value in Python is essentially an object, so the value here means an object; $A$ represents an attribute set which consists of attributes; $T$ represents all the types as well as the class hierarchy of these types ( $\preccurlyeq$ denotes the subtype relation); $\sigma$ denotes the value store mapping a variable or a heap location to a value; $\rho$ represents a type store mapping a variable or a heap location to a type; $\pi$ denotes an attribute set store mapping a value to an attribute set, representing the set of attributes of a value (or object); $\mathcal{F}_{\text {baset }}$ represents a function returning the default attribute set for a specific type; $\mathcal{F}_{\text {btype }}$ returns the type of a binary operation; $\mathcal{F}_{\text {index }}$ returns the index of the next iteration of a for-loop. The execution of a Python program in our system is a procedure of dynamically updating $T, \sigma, \rho$ and $\pi$.

Trace and Events. We model a few Python statements in our presentation shown in Fig. 4. Some of the symbols are defined in Fig. 3. For example, $\ell$ stands for a label that is similar to the source code line number. Observe that we model two specific predicates: the type check (isinst()) and the attribute check (hasattr()). The rest is standard.

An execution trace $\Gamma$ is a sequence of events recorded during the execution. An event is an execution instance of a statement, denoted as a tuple $\langle e i d, s,(v, \tau, A), \ell\rangle$ where eid represents a unique identifier; $s$ denotes the statement; $(v, \tau, A)$ represents the involved value, type and attribute set by the execution of the statement. Note that besides the value $v$, we also record the type and attribute set of each execution of the statement as they may be changed.

Tracing Rules. Table 1 represents the tracing rules for a set of commonly used Python statements. The first four rules specify four typical object creation statements. More specifically, rule (class-def) represents the tracing of a class definition statement, which creates and initializes a new class object. It creates a class object $o_{1}$ and associates it with $C$. It also creates a method object $o_{2}$, associated with the $m$ attribute of $o_{1}$. The attribute " $m$ " is added to the attribute set of the class object $o_{1}$. MethodType and Classtype are two built-in types in Python. CType and BType are symbols generated by our system to denote the types of class $C$ and $B$. As shown in the last column, the system generates two events $e_{1}$ and $e_{2}$ where $e_{1}$ represents the class object creation and $e_{2}$ represents the setup of the method in the class. 
Rule (func-def) specifies the rule for a function definition. It creates a function object and then assigns it to variable $f$. The corresponding trace event is straightforward. Rule (list-alloc) represents the tracing for creating a list object. It first creates a list object $o_{1}$ assigned to $y$ and then appends two elements to it $\left(o_{1} \_0\right.$ represents the first element). Accordingly, our system generates three events $e_{1}$, $e_{2}$ and $e_{3}$ with $e_{1}$ creating the list object, and $e_{2}$ and $e_{3}$ setting the elements. Rule (class-instant) represents object creation through class instantiation. It creates a new object $o_{1}$ and assigns it to $y$. The attribute set of $o_{1}$ is inherited from the object associated with the class $C$.

Rules (simple-assign), (bop-assign), (attr-read) and (attr-write) are standard. Particularly, the execution of an attribute write statement may dynamically change the attribute set of the object. Rule (for-loop) specifies the tracing for the next-iteration operation of a for-loop statement. The rule only specifies the situation in which the object is a collection (e.g., list, tuple). Rule (if-cmp), (ifisinst) and (if-hasattr) specify the tracing rules of three different kinds of branch predicates. In Rule (call), we record two events, one for parameter passing and the other for the call itself.

\subsection{Handling External Functions}

Function calls to external libraries are prevalent in Python programs. We cannot easily trace the execution of these libraries due to the lack of source code. However, if we do not trace their execution, the resulting trace is incomplete especially when these functions have side-effects on heap, leading to unsoundness of our later symbolic analysis. One way is to manually model the external functions. However, this requires tremendous efforts due to the sheer volume of these functions. Hence, our strategy is to manually model the commonly used external functions. For the others (which are still in a large number), we develop a technique to detect side-effects such that we handle them to avoid unsoundness. Next, we focus on discussing side-effect detection.

To detect side-effects, we maintain a value store $\theta$ : $\operatorname{Var} \mid$ HeapLoc $\mapsto$ Value recording the current value of each variable. The value store is only updated when the Python code is executed. In other words, it is not updated by execution of external functions. When a variable is used in the Python code, we compare the value from the store $\theta$ with the observed value from store $\sigma$. If the two are different, there must be side-effects by some external function call(s).

\begin{tabular}{|l|l|}
\hline & $v_{e} \leftarrow \sigma[\sigma[x] . f]$ \\
& $v_{r} \leftarrow \theta[\theta[x] . f]$ \#here, $v_{r}=1$ \\
1: x.f $=1$ & if $v_{e}=v_{r}:$ \\
2: y=extfunc (x) & $\Gamma \rightarrow \Gamma ;\langle z=x . f,(1, \ldots)\rangle$ \\
$3: \mathrm{z}=\mathrm{x} . \mathrm{f}$ & else $:$ \\
& $\Gamma \rightarrow \Gamma ;\left\langle x . f=v_{e},\left(v_{e}, \ldots\right)\right\rangle$ \\
& $\Gamma \rightarrow \Gamma ;\left\langle z=v_{e},\left(v_{e}, \ldots\right)\right\rangle$ \\
& $\theta \rightarrow \theta\left[z \mapsto v_{e}\right]$
\end{tabular}

Line 2 of the left column shows an unmodeled external function call, which has side-effects on $x$. When tracing the execution of line 3 , we use the rule shown in the right column. We first get the current value $v_{e}$ of the heap location pointed-to by $x . f$ from the execution environment $(\sigma)$, which is updated by both the Python code and the external function, and then query the recorded value of $x . f$ from $\theta$, which is only updated by Python code. If $v_{e}$ is equal to 1 , we treat it as an attribute read event. Otherwise, we concretize both the values of $x . f$ and $z$ due to the invisible side-effects that we cannot model, which essentially under-approximates the

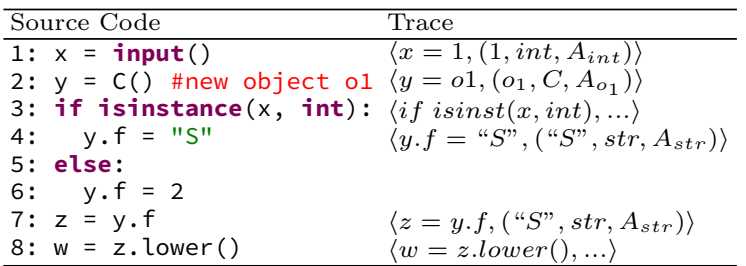

Figure 5: An Example Illustrating the Challenges

program state by fixing the input and output values of the external function. On the other hand, it ensures soundness.

\section{CONSTRAINT ENCODING}

Our technique is essentially a kind of predictive analysis $[4,5,6]$. As mentioned in Section 1, predictive analysis is based on the observed execution traces and can easily analyze the very long executions similar to the observed traces. Leveraging traces also allows mitigating the problems caused by dynamic features. While most existing predictive analyses explore different schedules of a concurrent execution and prohibit path changes, our technique explores the program state space caused by input variations, which may cause differences of execution paths and heap states. Such possible differences are encoded as constraints and an SMT solver is leveraged to explore the encoded state space to find bugs. Our technique is sound, meaning that it does not produce false positives ${ }^{2}$. Now we introduce the main challenges in encoding Python programs for predictive analysis.

(1) Dynamic typing in Python makes the type of a variable path sensitive, which means the same variable at the same program point may have different types depending on the execution path. However, symbolic variables in constraints are statically typed. Existing static encoding techniques often project a program variable (in its SSA form) to a symbolic variable in the constraint system. Such a method is no longer applicable. Note that the SSA form does not help in our context because even though the SSA form can express a variable having different types at different locations (by introducing multiple variables for each definition location), it cannot handle that a variable may have different types even at the same location. Note that the problem does not exist for symbolic execution or concolic execution as these techniques encode one path at a time.

(2) Besides encoding the value of a variable like in most existing work, we have to explicitly encode the type and the attribute set of a variable (or object) in order to reason about such features.

(3) Some bugs can only be triggered when the execution goes through a path different from the trace. Therefore, to predict such kind of bugs, we should also include the unexecuted branches into the constraint model.

Example. We use an example in Fig. 5 to illustrate the above challenges. The left column is the code snippet. Line 1 represents the initialization of $x$ which may accept a value of various types (e.g., int and string). Line 2 creates a new object of class $\mathrm{c}$, denoted as 01 . Line 3 is a type check predicate. If $x$ is of the int type, it writes a string " $\mathrm{s}$ " to the attribute "f" of o1. Otherwise it writes the integer 2. Note that, the initial attribute set of o1 does not contain the attribute " $f$ ", so the execution will dynamically add it to the attribute set of o1. Line 7 reads the attribute and

\footnotetext{
${ }^{2}$ We put the proof in our technical report: https://sites.google.com/ site/pypredictor/
} 
Table 1: Tracing Rules for Typical Statements

\begin{tabular}{|c|c|c|c|}
\hline Rule & Statement & Execution States & Events (eid and $\ell$ omitted) \\
\hline (class-def) & $\begin{array}{l}\text { class } C(B): \\
\text { def } m(\operatorname{sel} f, x): \\
\quad \text { self.x } x\end{array}$ & $\begin{aligned} & \sigma \rightarrow \sigma\left[C \mapsto o_{1}=\text { newobject }(),\right. \\
& o_{1} . m\left.\mapsto o_{2}=\text { newobject }()\right] \\
& \rho \rightarrow \rho[C \mapsto \text { ClassType } \\
& o_{1} . m\mapsto \text { MethodType }] \\
& \pi \rightarrow \pi\left[o_{1}\right. \mapsto \mathcal{F}_{\text {baset }}(\text { ClassType }) \cup\{" m "\}, \\
& o_{2}\left.\mapsto \mathcal{F}_{\text {baset }}(\text { MethodType })\right] \\
& T \rightarrow T \cup\{C \text { Type } \preccurlyeq \text { BType }\}\end{aligned}$ & $\begin{array}{l}e_{1}:\left\langle C=o_{1},\left(o_{1}, \sigma[C], \pi\left[o_{1}\right]\right)\right\rangle \\
e_{2}:\left\langle C \cdot m=o_{2},\left(o_{2}, \sigma\left[o_{1} \cdot m\right], \pi\left[o_{2}\right]\right)\right\rangle \\
\Gamma \rightarrow \Gamma ; e_{1} ; e_{2}\end{array}$ \\
\hline (func-def) & $\begin{array}{l}\text { def } f(x): \\
\quad \text { return } x\end{array}$ & $\begin{array}{l}\sigma \rightarrow \sigma\left[f \mapsto o_{1}=\text { newObject }()\right] \\
\rho \rightarrow \rho[f \mapsto \text { FuncType }] \\
\pi \rightarrow \pi\left[o_{1} \mapsto \mathcal{F}_{\text {baset }}(\text { FuncType })\right]\end{array}$ & $\Gamma \rightarrow \Gamma ;\left\langle f=o_{1},\left(o_{1}, \sigma[f], \pi\left[o_{1}\right]\right\rangle\right.$ \\
\hline (list-alloc) & $y=[1,2]$ & $\begin{array}{l}\sigma \rightarrow \sigma\left[y \mapsto o_{1}, o_{1-0} \mapsto 1, o_{1 \_} 1 \mapsto 2\right] \\
\rho \rightarrow \rho\left[y \mapsto \text { list }, o_{1-0} \mapsto \text { int }, o_{1-1} \mapsto \text { int }\right] \\
\pi \rightarrow \pi\left[o_{1} \mapsto \mathcal{F}_{\text {baset }}(\text { list })\right]\end{array}$ & $\begin{array}{l}e_{1}:\left\langle y=o_{1},\left(o_{1}, \text { list }, \mathcal{F}_{\text {baset }}(\text { list })\right)\right\rangle \\
e_{2}:\left\langle y \_0=1,\left(1, \text { int }, \mathcal{F}_{\text {baset }}(\text { int })\right)\right\rangle \\
e_{3}:\left\langle y \_1=2,\left(2, \text { int }, \mathcal{F}_{\text {baset }}(\text { int })\right)\right\rangle \\
\Gamma \rightarrow \Gamma ; e_{1} ; e_{2} ; e_{3}\end{array}$ \\
\hline (class-instant) & $y=c() \# c$ is a class & $\begin{array}{l}\sigma \rightarrow \sigma\left[y \mapsto\left(o_{1}=\text { newObject }()\right)\right] \\
\rho \rightarrow \rho[y \mapsto C T y p e] \pi \rightarrow \pi\left[o_{1} \mapsto \pi[\sigma[C]]\right.\end{array}$ & $\Gamma \rightarrow \Gamma ;\left\langle y=o_{1},\left(o_{1}\right.\right.$, CType,$\left.\left.\pi(C)\right)\right\rangle$ \\
\hline (simple-assign) & $y=x$ & $\sigma \rightarrow \sigma[y \mapsto \sigma(x)] \rho \rightarrow \rho[y \mapsto \rho(x)]$ & $\Gamma \rightarrow \Gamma ;\langle y=x,(\sigma(y), \rho(y), \pi(\sigma(y)))\rangle$ \\
\hline (bop-assign) & $z=x$ bop $y$ & $\begin{array}{l}\sigma \rightarrow \sigma[z \mapsto \sigma[x] \text { bop } \sigma[y]] \\
\rho \rightarrow \rho\left[z \mapsto \mathcal{F}_{\text {btype }}(\rho[x], \rho[y], \text { bop })\right] \\
\pi \rightarrow \pi\left[\sigma(z) \mapsto F_{\text {baset }}(\rho[z])\right]\end{array}$ & $\Gamma \rightarrow \Gamma ;\langle z=x$ bop $y,(\sigma[z], \rho[z], \pi[\sigma[z]])\rangle$ \\
\hline (attr-read) & $y=x \cdot f \mid y=\operatorname{getattr}\left(x, f^{\prime}\right)$ & $\sigma \rightarrow \sigma[y \mapsto \sigma[\sigma[x] . f]] \rho \rightarrow \rho[y \mapsto \rho[\sigma[x] . f]]$ & $\Gamma \rightarrow \Gamma ;\langle y=x . f,(\sigma[y], \rho[y], \pi[\sigma[y]])\rangle$ \\
\hline (attr-write) & $y \cdot f=x \mid \operatorname{setattr}\left(y, f^{\prime}, x\right)$ & $\begin{array}{l}\sigma \rightarrow \sigma[\sigma[y] . f \mapsto \sigma[x]] \rho \rightarrow \rho[\sigma[y] . f \mapsto \rho[x]] \\
\pi \rightarrow \pi[\sigma[y] \mapsto \pi[\sigma[y]] \cup\{" f "\}]\end{array}$ & $\Gamma \rightarrow \Gamma ;\langle y . f=x,(\sigma[x], \rho[x], \pi[x])\rangle$ \\
\hline (for-loop) & for $x$ in $y \ldots$ & $\begin{array}{l}i \leftarrow \mathcal{F}_{\text {index }}(\sigma[y]) \\
\sigma \rightarrow \sigma\left[x \mapsto \sigma\left[\sigma[y]_{\_} i\right]\right] \rho \rightarrow \rho\left[x \mapsto \rho\left[\sigma[y]_{-} i\right]\right]\end{array}$ & $\Gamma \rightarrow \Gamma ;\left\langle x=y_{-} i,(\sigma[x], \rho[x], \pi[\sigma[x]])\right\rangle$ \\
\hline (if-cmp) & if $(x$ relop $y) \ldots$ & $r \leftarrow(\sigma[x]$ relop $\sigma[y])$ & $\Gamma \rightarrow \Gamma ;\left\langle\operatorname{relop}(x, y),\left(r\right.\right.$, bool, $\mathcal{F}_{\text {baset }}($ bool $\left.\left.)\right)\right\rangle$ \\
\hline (if-isinst) & if (isinstance $(x$, list $)) \ldots$ & $r \leftarrow(\rho[x] \preccurlyeq$ list $)$ & $\Gamma \rightarrow \Gamma ;\left\langle\right.$ isinst $(x$, list $),\left(r\right.$, bool, $\mathcal{F}_{\text {baset }}($ bool $\left.\left.)\right)\right\rangle$ \\
\hline (if-hasattr) & if $\left(\right.$ hasattr $\left.\left(x, f^{\prime}\right)\right) \ldots$ & $r \leftarrow(" f " \in \pi[\sigma[x]])$ & $\Gamma \rightarrow \Gamma ;\left\langle\right.$ hasattr $(x$, "f" $),\left(r\right.$, bool, $\mathcal{F}_{\text {baset }}($ bool $\left.\left.)\right)\right\rangle$ \\
\hline (call) & $y=f(a)$ & $\begin{array}{l}\sigma \rightarrow \sigma[b \mapsto \sigma[a]] \rho \rightarrow \rho[b \mapsto \rho[a]] \\
(b \text { is the formal parameter })\end{array}$ & $\begin{array}{l}e_{1}:\langle b=a,(\sigma[a], \rho[a], \pi[\sigma[a]])\rangle \\
e_{2}:\langle y=f(a),(\sigma[\sigma[a] . f], \\
\left.\left.\text { FuncType, } \mathcal{F}_{\text {baset }}(\text { FuncType })\right)\right\rangle \Gamma \rightarrow \Gamma ; e_{1} ; e_{2}\end{array}$ \\
\hline
\end{tabular}

assigns it to $z$. Line 8 calls a method lower() of $z$. The bug will occur when the else branch is taken because the value of $z$ is of the int type and does not have the lower() method. However, the bug does not manifest itself when the true branch is executed.

From the example, we observe that $z$ at line 7 may have different types depending on the path and SSA cannot address the problem. To reason about the predicate at line 3 and the bug at line 8 , we need to explicitly encode the types and attribute sets. Assuming the trace is along the true branch, to expose the bug, we also need to encode the else branch, which may not be taken in the observed execution.

\subsection{Encoding Scheme}

Next we explain our encoding scheme from different aspects.

Using Multiple Symbolic Variables to Denote the Value of a Python Variable. We use multiple symbolic variables (of different types) to represent the value of a Python variable of different types. More specifically, given a Python variable $x$, we use a set of symbolic variables in the form of $\psi_{\tau}^{\sigma}$ to denote its value of type $\tau$. The superscript indicates this is a symbolic variable for value. For example, if $x$ can be an integer or a string, we use two symbolic variables $\psi_{\text {int }}^{\sigma}(x)$ and $\psi_{\text {str }}^{\sigma}(x)$ to denote its value. For a statement $s$ involving $x$, both symbolic variables are updated to encode the possible behavior of $s$ when $x$ has different types.

Explicitly Reasoning about Types. To encode the dynamic typing behavior, we introduce a new symbolic variable $\psi^{\rho}(x)$ to represent the type of the variable $x$. The superscript indicates this is a variable for type. We support equivalence and non-equivalence checking, and sub-typing on the values of these variables. We encode the subtype relation as a partial order between types, which is supported by the solver.

Explicitly Reasoning about Attributes. We introduce symbolic variable $\psi^{\pi}(o b j)$ to represent the attribute-set of an object $o b j$. Note that the attribute sets are associated with objects instead of variables following the Python memory model. In particular, if $x$ and $y$ are aliases through the assignment $y=x$, then the statement $x . f=\ldots$ adds the attribute " $f$ " to the object that both $x$ and $y$ can reference. We represent the attribute set as a set in the constraint system and support the membership operation.

Encoding Rules. Before encoding, we first transform the trace into its single static assignment (SSA) form so that every variable is defined exactly once. We use $x^{i}$ to denote the definition instance of $x$ at trace entry $i$. We also extend the trace with statements from unexecuted branches (see Section 5.2). As such, $\phi$-functions are also introduced to integrate definitions from two branches of a conditional statement at the join point. Table 2 shows the encoding rules for the typical events.

Rule (1) specifies the encoding for the assignment with a constant. The symbolic value variable $\psi_{\tau}^{\sigma}\left(z^{i}\right)$ is asserted to the value $v$. The symbolic type variable is asserted to type $\tau$, and the object created to represent the value of $z^{i}$, which is acquired by a function $\operatorname{obj}\left(z^{i}\right)$, is asserted to have the attribute set $A$.

Rule (2) specifies the encoding for a simple assignment. In the encoding, since we introduce a set of symbolic variables of different types to denote the value of a program variable of various types, for each type $\tau$, we assert the equivalence of the corresponding (symbolic) value of $z^{i}$ and $x^{j}$. This rule does not affect the attribute set, which is associated with the object instead of the variable.

Rule (3) encodes the addition operation. The basic idea is that for each type having the addition operation, we encode its behavior. For simplicity, we assume the solver only supports additions for int and string types. As such, the first two clauses assert integer addition and string concatenation, respectively. The third one encodes additions that are not supported by the solver such that we cannot reason 
Table 2: Encoding Rules. The events are in the SSA form with the superscript of a variable $x$ representing the id of the event where $x$ is most recently defined. $\operatorname{obj}(x)$ represents the object created for variable $x . \psi^{\sigma}(x), \psi^{\rho}(x)$, and $\psi^{\pi}(x)$ represent the symbolic variables denoting value, type, and attribute set for a program variable/value $x$. Each type $\tau$ means each possible type of $x$. type $(o)$ denotes the type of object $o$.

\begin{tabular}{|c|c|}
\hline SSA Formed Event $\left(e_{i}\right)$ & Encoded Symbolic Constraints \\
\hline$(1)\left\langle z^{i}=v,(v, \tau, A)\right\rangle$ & $\psi_{\tau}^{\sigma}\left(z^{i}\right)=v \wedge \psi^{\rho}\left(z^{i}\right)=\tau \wedge \psi^{\pi}\left(o b j\left(z^{i}\right)\right)=A$ \\
\hline$(2)\left\langle z^{i}=x^{j}, \ldots\right\rangle$ & $\left(\wedge_{\text {each type } \tau} \psi_{\tau}^{\sigma}\left(z^{i}\right)=\psi_{\tau}^{\sigma}\left(x^{j}\right)\right) \wedge \psi^{\rho}\left(z^{i}\right)=\psi^{\rho}\left(x^{j}\right)$ \\
\hline (3) $\left\langle z^{i}=x^{j}+y^{k},(v, \tau, A)\right\rangle$ & $\begin{array}{l}\text { if }\left(\psi^{\rho}\left(x^{j}\right)=\operatorname{int} \wedge \psi^{\rho}\left(y^{k}\right)=\text { int }\right) \Rightarrow\left(\psi_{\text {int }}^{\sigma}\left(z^{i}\right)=\psi_{\text {int }}^{\sigma}\left(x^{j}\right)+\psi_{\text {int }}^{\sigma}\left(y^{k}\right) \wedge \psi^{\rho}\left(z^{i}\right)=\operatorname{int} \wedge \psi^{\pi}\left(\operatorname{obj}\left(z^{i}\right)\right)=A_{\text {int }}\right) \\
\rangle \text { elif }\left(\psi^{\rho}\left(x^{j}\right)=\operatorname{str} \wedge \psi^{\rho}\left(y^{k}\right)=\operatorname{str}\right) \Rightarrow\left(\psi_{\text {str }}^{\sigma}\left(z^{i}\right)=\operatorname{concat}\left(\psi_{\text {str }}^{\sigma}\left(x^{j}\right), \psi_{\text {str }}^{\sigma}\left(y^{k}\right)\right) \wedge \psi^{\rho}\left(z^{i}\right)=\operatorname{str} \wedge \psi^{\pi}\left(\operatorname{obj}\left(z^{i}\right)\right)=A_{\text {str }}\right) \\
\text { else }\left(\psi_{\tau}^{\sigma}\left(z^{i}\right)=v \wedge \psi^{\rho}\left(z^{i}\right)=\tau \wedge \psi^{\pi}\left(\operatorname{obj}\left(z^{i}\right)\right)=A\right) \wedge \psi_{\text {type }\left(\operatorname{obj}\left(x^{j}\right)\right)}^{\sigma}\left(x^{j}\right)=\operatorname{obj}\left(x^{j}\right) \wedge \psi_{\text {type }\left(\operatorname{obj}\left(y^{k}\right)\right)}^{\sigma}\left(y^{k}\right)=\operatorname{obj}\left(y^{k}\right) \\
\end{array}$ \\
\hline (4) $\left\langle i f i \operatorname{sinst}\left(x^{j}, \tau\right), \ldots\right\rangle$ & $\psi^{\rho}\left(x^{j}\right) \preccurlyeq \tau$ \\
\hline (5) $z^{i}=\phi\left(e_{k}, z^{t}, z^{f}\right)$ & $\begin{array}{l}\Phi\left(e_{k}\right) \Rightarrow\left(\left(\bigwedge_{\text {each type } \tau} \psi_{\tau}^{\sigma}\left(z^{i}\right)=\psi_{\tau}^{\sigma}\left(z^{t}\right)\right) \wedge \psi^{\rho}\left(z^{i}\right)=\psi^{\rho}\left(z^{t}\right)\right) \wedge \\
\neg \Phi\left(e_{k}\right) \Rightarrow\left(\left(\bigwedge_{\text {each type } \tau} \psi_{\tau}^{\sigma}\left(z^{i}\right)=\psi_{\tau}^{\sigma}\left(z^{f}\right)\right) \wedge \psi^{\rho}\left(z^{i}\right)=\psi^{\rho}\left(z^{f}\right)\right)\end{array}$ \\
\hline (6) $\left\langle y^{i}=x^{j} \cdot f, \ldots\right\rangle$ & $\bigwedge_{o \in p t s(x)}\left(\psi_{\text {type }(o)}^{\sigma}\left(x^{j}\right)=o\right) \Rightarrow\left(\left(\bigwedge_{\text {each type } \tau} \psi_{\tau}^{\sigma}\left(y^{i}\right)=\psi_{\tau}^{\sigma}\left(o . f^{k}\right)\right) \wedge \psi^{\rho}\left(y^{i}\right)=\psi^{\rho}\left(o . f^{k}\right)\right)$ \\
\hline (7) $\left\langle y^{j} \cdot f=x^{k}, \ldots\right\rangle$ & $\begin{aligned} &\left(\psi_{\text {type }(o)}^{\sigma}\left(y^{j}\right)=o\right) \Rightarrow\left(\left(\bigwedge_{\text {each type } \tau} \psi_{\tau}^{\sigma}\left(o . f^{i}\right)=\psi_{\tau}^{\sigma}\left(x^{k}\right)\right) \wedge \psi^{\rho}\left(o . f^{i}\right)=\psi^{\rho}\left(x^{k}\right) \wedge \psi^{\pi}(o)^{\prime}=\psi^{\pi}(o) \cup\left\{“ f^{\prime \prime}\right\}\right) \wedge \\
& \bigwedge_{o \in p t s\left(y^{j}\right)}\left(\psi_{\text {type }(o)}^{\sigma}\left(y^{j}\right) \neq o\right) \Rightarrow\left(\left(\bigwedge_{\text {each type } \tau} \psi_{\tau}^{\sigma}\left(o . f^{i}\right)=\psi_{\tau}^{\sigma}\left(o . f^{p}\right)\right) \wedge \psi^{\rho}\left(o . f^{i}\right)=\psi^{\rho}\left(o . f^{p}\right) \wedge \psi^{\pi}(o)^{\prime}=\psi^{\pi}(o)\right)\end{aligned}$ \\
\hline (8) 〈if hasattr $\left(x^{j}, "\right.$ " $\left.\left.f "\right), \ldots\right\rangle$ & $\cdot\rangle_{o \in p t s\left(x^{j}\right)}\left(\psi_{\text {type }(o)}^{\sigma}\left(x^{j}\right)=o\right) \wedge\left(" f " \in \psi^{\pi}(o)\right)$ \\
\hline
\end{tabular}

about their value variations. We assert the value, type, attribute set of $z^{i}$ to the observed ones. More importantly, we also need to assert the operands $x^{j}$ and $y^{k}$ must hold their observed values to ensure soundness.

Rule (4) encodes a type check predicate. It simply asserts the type of the variable is a subclass of the intended type $\tau$.

Rule (5) specifies the encoding for the $\phi$ function that joins the updates of a variable in the two branches of a predicate: $z^{i}$ is equal to $z^{t}$ if the predicate $e_{k}$ holds, and is equal to $z^{f}$ otherwise, where $z^{t}$ and $z^{f}$ are the most recent versions of $z$ inside the branches. In the encoding, all the symbolic value and type variables are updated according to the encoding of $e_{k}$, denoted as $\Phi\left(e_{k}\right)$. The encoding of object attribute sets is also handled path-sensitively, similar to $\phi$ functions for variables. In other words, we also join the attribute sets of an object in a path sensitive fashion at a merge point of branches. Details are elided.

Rule (6) encodes an object attribute read. The encoding relies on a pre-computed points-to set $p t s\left(x^{j}\right)$ representing the set of objects that may be pointed to by $x^{j}$. We use the Anderson algorithm [10] to compute the may points-to set on the trace (extended with the un-executed branches). Intuitively, our extended trace can be considered as a program with loop unrolled and dynamic features uncovered. In the encoding, for the object $o$ in the points-to set that $x^{j}$ actually points-to, we assert the equivalence of the values of $o . f^{k}$ and $y^{i}$ and the equivalence of their types. Note that the superscript $k$ denotes the most recent definition point of o.f. Function type (o) represents the type of object $o$.

Rule (7) encodes an object attribute write. For the object $o$ pointed-to by $y^{j}$, we assert the equivalence of both the values and types of $o . f^{i}$ and $x^{k}$. Besides, we assert the new attribute set is the union of the old attribute set and the " $f$ ". If the object is not pointed to by $y^{j}$, we assert the value and type, and the attribute-set of the base object stay the same. Note that the superscript $i$ and $p$ denote the current and the most recent definition points of the object attribute, respectively. We use $\psi^{\pi}(o)^{\prime}$ to denote a new defined version of the symbolic attribute set of $o$.

Rule (8) encodes an attribute check. For any object $o$ in the points-to set of $x^{j}$, if the object referred to by $x^{j}$ equals $o$, the attribute " $f$ " must be in the attribute set of $o$.
Handling Solver Unsupported Types. Python supports many more types compared to those supported by the solver. In our encoding rules, we do not specify the details of handling solver unsupported types for space reason. In fact, we treat the values of solver unsupported types as objects denoted by IDs. We only reason about equivalence and nonequivalence of object IDs. This is consistent with existing work of predictive analysis $[8,7]$.

\subsection{Unexecuted Path Relaxation}

To allow reasoning about possible path changes induced by input variations, we need to include the unexecuted branches to our constraint system. The basic idea is to enhance the observed trace to aggressively include statements in unexecuted branches as long as those statements can be fully resolved by static analysis. We preclude the unresolved branches by asserting the branch outcome as the observed one. This ensures the soundness of our analysis, although it limits the coverage. Next, we explain how we determine if an unexecuted statement can be resolved.

Assignments. Constant assignments $x=v$ can always be resolved and encoded. For a simple assignment $y=x$, our analysis first looks for the most recent definition of $x$. If it may be defined in an unresolved branch, which was hence not encoded, we consider the variable $x$ unresolved. As a result, the assignment and the whole branch are also unresolved. Assignments of binary operations like $z=x+y$ are handled similarly.

Conditionals and Loops. For a conditional statement if $x>$ $y s_{1}$ else $s_{2}$ inside an unexecuted branch, we first try to resolve $x$ and $y$ and then $s_{1}$ and $s_{2}$. If any of these cannot be resolved, the conditional is not resolved, except the following case. If $x$ and $y$ can be resolved, and one of the branches can be resolved, say $s_{1}$. We encode $s_{1}$ and assert the branch outcome to be true. For a loop statement in an unexecuted branch, we require the loop bound can be statically resolved as constant.

Calls. For a function call in an unexecuted branch, we must ensure it is not a recursive call or an unmodeled external function. If this is satisfied and the callee can be resolved, we say the invocation can be resolved. If a call statement has multiple possible callees and only some of them can be 


\begin{tabular}{ll}
\hline Source Code & Trace(Instruction) \\
\hline $1: \mathrm{x}=[1,2]$ & $10: x^{1}=[1,2]$ \\
$2: \mathrm{y}=[3]$ & $11: y^{2}=[3]$ \\
3:if $\mathrm{a}>\odot:$ & $12:$ if $a^{0}>\odot:$ \\
$4: \mathrm{x}=\mathrm{y}$ & $13: x^{4}=y^{2}$ \\
$5:$ if $\mathrm{b}>\odot:$ & $14: x^{5}=\phi\left(a>0, x^{1}, x^{4}\right)$ \\
6: $\mathrm{y}=\mathrm{x}$ & $16: \mathrm{b}<=\odot$ \\
$7:$ for $\mathrm{i}$ in $\mathrm{y}:$ & $17: z^{8}=y^{2}$ \\
$8: \quad \mathrm{t}=\mathrm{i}$ & \\
9: $\mathrm{z}=\mathrm{y}$ & \\
Figure 6: Unexecuted Path Relaxation.
\end{tabular}

resolved, we assert the call target falls into the set that can be resolved. We do not handle try-except statements. If we encounter any such statements in an unexecuted branch, the branch is considered unresolved.

Example. Consider the example in Fig. 6. The left column is the source code and the shaded statements are executed by the observed run. Lines 4 and $6-8$ are not executed. The right column shows the intermediate SSA form of the trace after we extend it with the unexecuted branches. Note that we are able to resolve and add line 4 as $y$ can be resolved. As a result, a $\phi$ statement is added at line 14. In contrast, the branch consisting of lines $6-8$ cannot be resolved as we cannot statically resolve the loop bound $y$ at line 7 . As such, we assert the branch outcome of line 5 to be false.

\subsection{Constraint Solving for Bug Detection}

After the extended trace is fully encoded, we further add assertions for bug detection before the constraints are passed to the solver. While the user can come up various customized bug detection assertions. In our current system, we mainly focus on two kinds of assertions.

Subtype Assertion. Subtype assertion is used to detect type errors. More specifically, we assert if the type of a variable may fall out of an acceptable range. For example, suppose the acceptable types of variable $x$ include int or str. We assert $\neg\left(\psi^{\rho}(x) \preccurlyeq i n t \vee \psi^{\rho}(x) \preccurlyeq s t r\right)$. For operations involving multiple variables, we check the type combinations of these variables. For example, for a binary operation $z=$ $x+y$, the Python interpreter does not allow the addition of a string and a number. Therefore, we assert $x$ is an integer and $y$ is string or vice versa.

Attribute Assertion. Attribute assertion is used to check if a non-existent attribute may be read. More specifically, for an attribute read $y=x . f$, let points-to set $p t s(x)=$ $\left\{o b j_{1}, \ldots, o b j_{n}\right\}$, we check if there exists $o b j_{t} \in p t s(x)$ that satisfies: (1) there is a feasible path making $x=o b j_{t}$ satisfiable, and (2) $o b j_{t}$ does not contain the attribute " $f$ ".

We send all the constraints to the solver. If the result is SAT, we find a bug and its triggering input.

\section{EVALUATION}

We have implemented a prototype in Python. The system mainly consists of a tracing infrastructure and a constraint encoding and solving component. As mentioned in earlier sections, Python programs tend to use a lot of external functions and classes implemented in other languages. We manually modeled a set of commonly used external functions by rewriting them in Python. We use Z3 as the SMT solver. To improve scalability, we also implement a dynamic slicer to prune unrelated trace entries for a given assertion. We make our prototype along with the evaluation environment publically available at [27].
Our evaluation aims to address the following questions:

RQ1: How effective is our approach in detecting potential bugs for real world Python projects?

RQ2: How efficient is our approach? Can it scale to real world programs and executions?

RQ3: How applicable is it to use the test cases provided in the projects as the observed runs? Can our approach detect more bugs by increasing test coverage?

To answer the above research questions, we apply our approach to a set of real world Python projects, as shown in Table 3 (columns 1-2). These benchmarks are mainly from GitHub. All of them are highly ranked in popularity and widely used in practice. To select these benchmarks, we went through the ranking of popularity and bug reports, and selected those that have the highest ranking and at least one reproducable bug report. We also considered diversity during selection. Observe that some of them are large. The largest one has over $84 \mathrm{~K}$ source lines of Python code. The sum of the source code lines of these projects is about $164 \mathrm{~K}$.

These projects fall in the following application domains, which are representative for Python programs:

Requests and urllib3 - very popular HTTP libraries.

Fabric and salt - well-known remote deployment and system administration applications.

Flask, bottle and web2py - widely used Web application development frameworks.

Tweepy, boto and praw - well known web service APIs. Bs4 - a very popular HTML and XML parsing library. Our experiments were conducted on a machine with an Intel i5-4210U, 4GB RAM, Ubuntu 14.04, and Python 2.7.4.

\subsection{Experimental Results}

Table 3 shows the experimental results. For each program, we first run the (passing) test cases provided as part of the project and then encode these traces together with unexecuted branches to find bugs. In Table 3, we only report the statistics for those test runs that allow us to identify bugs.

Trace and Constraints. Column 3 (Mod) presents the number of modules executed, which ranges from 2 to 36. Note that we have excluded some uninteresting modules such as those related to logging and multiprocessing. Columns 4-7 report the number of instructions that fall into some popular categories, namely, attribute reads (AttrR), attribute writes (AttrW), predicates (Pred) and calls. Column 8 presents the total number of events in the trace. Observe that many of these executions are long. The longest execution has over $70 \mathrm{~K}$ events.

Column 9 (AP) presents the number of events encoded when reaching the buggy assertion point. Recall that our system adds assertions in order to find bugs (Section 5.3). Column 10 (ExtE) presents the number of unexecuted statements that are added to the encoding in column 9. Observe that our technique was able to resolve a lot of unexecuted branches, allowing us to explore a large number of neighboring executions of the observed run. Column 11 (Cons) presents the total number of constraints when reaching the assertion of the violation, which ranges from 41 to over $135 \mathrm{~K}$. Detected Defects (RQ1). Our system is able to detect a set of bugs for these programs. The reported bugs are all true positives. Column 12 presents the bug issue ids if available. The issue ids prefixed with ${ }^{*}$ are the new bugs that have not been reported before. We reported these new defects to the developers through the bug tracker of Github 
Table 3: Statistics of Experimental Results

\begin{tabular}{|c|c|c|c|c|c|c|c|c|c|c|c|c|c|c|c|}
\hline \multicolumn{2}{|c|}{ (a)Benchmarks } & \multicolumn{6}{|c|}{ (b) Trace } & \multicolumn{3}{|c|}{ (c)Constraints } & \multicolumn{2}{|c|}{ (d)Defects } & \multicolumn{3}{|c|}{ (e)Time(sec) } \\
\hline Name & $\begin{array}{l}\text { SLOC } \\
\end{array}$ & Mod & AttrR & AttrW & Pred & Calls & Events & $\mathrm{AP}$ & ExtE & Cons. & Issue\# & Count & Tracing & Solving & Total \\
\hline \multirow[t]{12}{*}{ requests } & 9934 & 36 & 1257 & 2506 & 1022 & 1331 & 18558 & 6468 & 1223 & 11461 & 1462 & $8+4$ & 6.89 & 84.74 & 91.63 \\
\hline & & 8 & 126 & 37 & 72 & 107 & 982 & 71 & 0 & 144 & 2077 & & 0.62 & 0.83 & 1.45 \\
\hline & & 35 & 1680 & 3566 & 1537 & 1738 & 26436 & 8107 & 1445 & 14378 & 1711 & & 9.31 & 96.08 & 105.39 \\
\hline & & 31 & 1159 & 2360 & 968 & 1230 & 17225 & 11980 & 2494 & 20965 & 1082 & & 5.12 & 121.52 & 126.64 \\
\hline & & 30 & 1350 & 10097 & 1108 & 1406 & 42092 & 7309 & 1318 & 12886 & 1324 & & 8.3 & 78.96 & 87.26 \\
\hline & & 33 & 1285 & 10192 & 1078 & 1320 & 41707 & 10656 & 2201 & 18669 & 1019 & & 8.03 & 106.71 & 114.74 \\
\hline & & 34 & 975 & 2449 & 830 & 1155 & 15943 & 7852 & 1460 & 13763 & 2613 & & 11.9 & 98 & 109.9 \\
\hline & & 10 & 86 & 223 & 101 & 160 & 1801 & 71 & 5 & 139 & 2267 & & 1.27 & 1.87 & 3.14 \\
\hline & & 11 & 140 & 258 & 144 & 196 & 1257 & 2819 & 651 & 4887 & $*_{2527}$ & & 0.97 & 29.1 & 30.07 \\
\hline & & 12 & 266 & 353 & 232 & 330 & 3708 & 4467 & 878 & 7910 & $* 2552$ & & 1.59 & 42.03 & 43.62 \\
\hline & & 29 & 902 & 1942 & 782 & 1059 & 13623 & 6734 & 1261 & 11781 & $*_{2638}$ & & 6.31 & 84.4 & 90.71 \\
\hline & & 35 & 982 & 2348 & 834 & 1161 & 15666 & 2824 & 534 & 5093 & *2639 & & 6.64 & 40.4 & 47.04 \\
\hline \multirow[t]{2}{*}{ urllib3 } & 4798 & 7 & 41 & 38 & 20 & 43 & 406 & 168 & 15 & $\begin{array}{r}324 \\
\end{array}$ & 355 & 2 & 0.31 & 1.52 & 1.83 \\
\hline & & 10 & 347 & 8695 & 310 & 387 & 28801 & 505 & 69 & 836 & 143 & & 4.29 & 6.88 & 11.17 \\
\hline \multirow[t]{7}{*}{ fabric } & 2995 & 10 & 188 & 143 & 179 & 169 & 2318 & 289 & 33 & 609 & 1204 & $6+1$ & 1.61 & 4.2 & 5.81 \\
\hline & & 6 & 31 & 25 & 13 & 32 & 302 & 243 & 17 & 482 & 610 & & 0.24 & 3.32 & 3.56 \\
\hline & & 2 & 6 & 0 & 4 & 7 & 57 & 30 & 3 & 55 & 1096 & & 0.06 & 0.69 & 0.75 \\
\hline & & 4 & 6 & 0 & 5 & 7 & 57 & 30 & 3 & 55 & 1096 & & 0.07 & 0.63 & 0.7 \\
\hline & & 3 & 20 & 14 & 14 & 11 & 168 & 111 & 18 & 195 & 1191 & & 0.28 & 1.24 & 1.52 \\
\hline & & 2 & 9 & 0 & 8 & 6 & 71 & 57 & 15 & 100 & 1096 & & 0.08 & 0.83 & 0.91 \\
\hline & & 3 & 21 & 14 & 15 & 16 & 171 & 117 & 23 & 196 & *1303 & & 0.15 & 1.64 & 1.79 \\
\hline \multirow[t]{13}{*}{ salt } & 84789 & 13 & 1264 & 330 & 594 & 863 & 7341 & 12667 & 5368 & 17978 & 5959 & $6+7$ & 12.26 & 49.85 & 62.11 \\
\hline & & 13 & 2438 & 2806 & 1970 & 2058 & 30099 & 10930 & 2436 & 18988 & 11873 & & 13.3 & 106.91 & 120.21 \\
\hline & & 14 & 1298 & 356 & 630 & 903 & 7736 & 12869 & 5432 & 18327 & 12651 & & 7.15 & 89.9 & 97.05 \\
\hline & & 5 & 19 & 11 & 11 & 15 & 160 & 23 & 2 & 41 & 6817 & & 0.49 & 0.35 & 0.84 \\
\hline & & 19 & 2296 & 694 & 1320 & 1533 & 15385 & 13122 & 5436 & 18837 & 12453 & & 9.76 & 90.1 & 99.86 \\
\hline & & 4 & 9 & 25 & 11 & 27 & 198 & 227 & 29 & 398 & 8635 & & 0.4 & 2.42 & 2.82 \\
\hline & & 14 & 1303 & 358 & 632 & 906 & 6696 & 12781 & 5290 & 18184 & $*_{22547}$ & & 6.99 & 88.66 & 95.65 \\
\hline & & 14 & 1341 & 306 & 644 & 942 & 7728 & 12515 & 5359 & 17689 & $*^{*} \mathrm{~s} 22547$ & & 9.55 & 109.24 & 118.79 \\
\hline & & 15 & 1344 & 304 & 648 & 927 & 7686 & 12635 & 5414 & 17834 & $* 24820$ & & 11.36 & 122.27 & 133.63 \\
\hline & & 15 & 1817 & 904 & 1148 & 1678 & 15291 & 17563 & 6540 & 26468 & ${ }^{*} 25006$ & & 10.47 & 129.33 & 139.8 \\
\hline & & 15 & 1818 & 904 & 1149 & 1678 & 15295 & 14110 & 5566 & 20702 & *s25006 & & 10.99 & 106.3 & 117.29 \\
\hline & & 15 & 1817 & 904 & 1149 & 1678 & 15290 & 17571 & 6540 & 26486 & *s25006 & & 9.64 & 128.11 & 137.75 \\
\hline & & 19 & 1591 & 599 & 837 & 1433 & 11678 & 15398 & 6028 & 22692 & *s25006 & & 11.11 & 156.91 & 168.02 \\
\hline \multirow[t]{2}{*}{ flask } & 2645 & 9 & 2206 & 1014 & 1650 & 941 & 15975 & 21497 & 5696 & 33615 & 857 & 2 & 13.01 & 351.72 & 364.73 \\
\hline & & 25 & 10142 & 2456 & 4320 & 2333 & 70361 & 76215 & 8210 & 135908 & 237 & & 67.23 & 1549.81 & 1617.04 \\
\hline bottle & 3003 & 4 & 150 & 104 & 99 & 156 & 1625 & 1468 & 214 & 2680 & 223 & 1 & 0.71 & 18.03 & 18.74 \\
\hline \multirow[t]{2}{*}{ web2py } & & 2 & 16 & 13 & 8 & 14 & 125 & 48 & 7 & 80 & $11 / 01 / 12$ & $1+1$ & 1.28 & 1.32 & 2.6 \\
\hline & & 2 & 5 & 6 & 11 & 7 & 80 & 68 & 17 & 101 & *968 & & 3.97 & 1.29 & 5.26 \\
\hline \multirow[t]{2}{*}{ tweepy } & 2394 & 19 & 68 & 198 & 69 & 112 & 1316 & 1483 & 218 & 2748 & $12 / 22 / 13$ & $1+1$ & 1.25 & 26.9 & 28.15 \\
\hline & & 7 & 9695 & 1840 & 3707 & 5284 & 61571 & 67406 & 5899 & 121255 & $* 642$ & & 36.25 & 1165.21 & 1201.46 \\
\hline boto & 47904 & 18 & 265 & 265 & 158 & 45 & 2619 & 2766 & 535 & 4840 & $03 / 19 / 12$ & 1 & 3.07 & 70.19 & 73.26 \\
\hline praw & 3034 & 4 & 87 & 152 & 86 & 68 & 1324 & 19 & 4 & 38 & 87 & 1 & 7.22 & 0.4 & 7.62 \\
\hline \multirow[t]{3}{*}{ bs 4} & 2718 & 7 & 41 & 42 & $\begin{array}{ll}77 \\
\end{array}$ & 79 & 771 & 44 & 10 & 79 & 1307471 & $1+2$ & 0.53 & 0.68 & 1.21 \\
\hline & & 5 & 83 & 50 & 101 & 83 & 921 & 171 & 47 & 254 & *1463984 & & 0.64 & 3.42 & 4.06 \\
\hline & & 5 & 66 & 42 & 81 & 75 & 783 & 160 & 44 & 244 & *1463984 & & 1.34 & 3.17 & 4.51 \\
\hline
\end{tabular}

or launchpad. Most of them have been confirmed and even fixed. Id "*s22547" means that the root cause of the bug is very similar to that of issue " 22547 ". There are three known bugs that we cannot find the issue numbers. Instead, we provide the commit records. Column 13 presents the number of the detected defects. Note that our approach is effective, identifying 46 defects with 30 known and 16 new.

Analysis Time (RQ2). Columns 14-16 present the tracing time, solving time, and total time, respectively. The solving time is the sum of the many queries to the solver regarding the bug identification assertions. From the results, we argue that the cost of our approach is reasonable. It detects most of these defects in a few seconds to a few minutes. The most time-consuming case is issue 237 of flask, which took about 27 minutes to process. Further inspection shows that it is due to the long execution trace.

Fault Detection Capability versus Test Coverage (RQ3). The detection capability of our approach largely depends on the observed runs. In our experiment, we mainly choose the test cases along with the projects as the seed runs. The reason is that test-driven development is pervasive in programming with dynamic languages. As such, there are often many test cases along with Python projects.

We randomly choose 100 passing test cases from the testsuites of two benchmarks, requests and salt, which have the largest number of defects. We then study how the accumulative number of detected bugs grows with the test coverage. Fig. 7(a) shows the result. Note that here we measure statement coverage. The result reveals a close-to-linear relation between the two. We hence argue that our approach can detect more defects with more test cases to achieve better coverage. In some sense, these test cases drive the execution to interesting places and our technique reasons about bugs in the neighboring executions. Fig. 7(b) shows the accumulative analysis time of our system. Observe that the growth is steady and the total time is reasonable.

\subsection{Defect Study}

To better understand the common patterns of defects in Python programs, we further examine these reported bugs and classify them into three categories as shown in Table 4. Attribute Errors. 23(50\%) of the detected defects cause AttributeError crashes, which mean that the execution accesses a nonexistent attribute. In Python programs, it is possible that an attribute is added to the object in one path but not in another path. Developers may forget the latter path and access the attribute which does not exist.

More specifically, there are 6 such defects in requests with 2 new defects. Consider the new issue 2527. PreparedRequest.copy () invokes the copy () method of object self._cookies, which is passed from some high level API (e.g., the instantiation method of class Request). Unfortunately, some of these APIs may not add the copy () method to the attribute set. There are 2 attribute defects in fabric. For instance, in issue 610, the key_filename () function of the network.py module checks if env.key_filename is an 
(a) Coverage and Defects

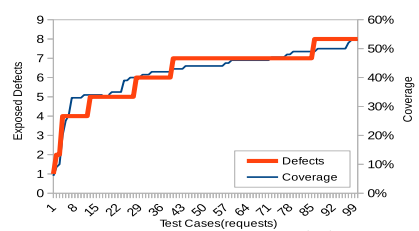

(b) Time Overhead
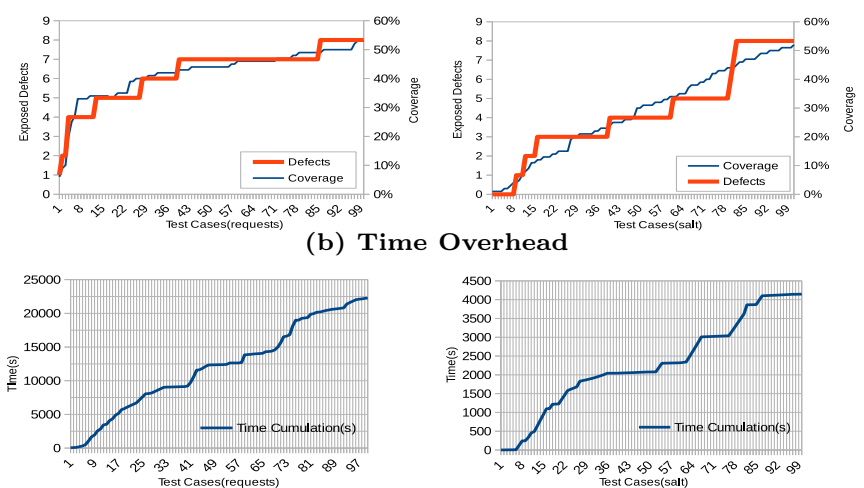

Figure 7: Bug Detecting Capability along with Coverage

instance of list type. If not, it will wrap env.key_filename into a list. Later on, it reads the "startswith" attribute of each item in the list. Our approach finds that the program will crash with an AttributeError raised when env.key_filename is initialized as another iterable type object (e.g., a set). This is because the program wraps the set object into a list and reads the "startswith" attribute of the set object, which does not exist.

Type Errors. $3(6.52 \%)$ of the detected defects cause TypeError crashes, which occur when a parameter of an invalid type is passed to a built-in function or when a binary operation has unmatched operands. The type error bug in requests is shown in Fig. 1 and discussed. The bug in salt occurs if the parameter passed to time.sleep () is not of a number type (e.g., int and float).

Unicode Errors. $20(43.48 \%)$ of the reported defects cause UnicodeEncodeError or UnicodeDecodeError crashes. Unicode errors are very common in Python applications, especially in Web related applications. The Python 2.x interpreter implicitly casts a byte string to a unicode string during certain operations (e.g., concatenation between a byte string and a unicode string). However, such casting crashes if the input string contains non-ascii characters. Specifically, we have found 5 such bugs in requests, and 2 of them are new bugs. In issue 1082, requests provides a set of HTTP APIs (e.g., get/put) that support values of different types for the parameters headers and data. Both parameters allow unicode and byte strings. The program crashes when headers contains a unicode string (e.g., headers $=\left\{u^{\prime}\right.$ "Content - Type": ... $\}$ ) and data contains a byte string with non-ascii characters. This is because an internal method_send_output() of module httplib.py concatenates them, triggering the implicit cast and causing the crash. We have also found unicode bugs in salt, all of which are not reported before.

\section{RELATED WORK}

Our approach belongs to the category of predictive analysis $[4,5,8]$. Existing predictive analyses were originally used in concurrency program analysis, such as race detection $[4,5,6,7]$ and atomicity violation detection [8]. They focus on changing the schedules to explore different executions. Differently, our approach is the first to change inputs to explore different executions. Besides, our approach allows the exploration of the unexecuted branches, while existing
Table 4: Classifications of Detected Defects

\begin{tabular}{|l|r|r|r|r|r|r|}
\hline \multirow{2}{*}{ Benchmarks } & \multicolumn{2}{|c|}{ AttributeError } & \multicolumn{2}{c|}{ TypeError } & \multicolumn{2}{c|}{ UnicodeError } \\
\cline { 2 - 7 } & Known & New & Known & New & Known & New \\
\hline requests & 4 & 2 & 1 & 0 & 3 & 2 \\
\hline urllib3 & 2 & 0 & 0 & 0 & 0 & 0 \\
\hline fabric & 2 & 0 & 1 & 0 & 3 & 1 \\
\hline salt & 5 & 1 & 1 & 0 & 0 & 6 \\
\hline flask & 1 & 0 & 0 & 0 & 1 & 0 \\
\hline bottle & 1 & 0 & 0 & 0 & 0 & 0 \\
\hline web2py & 0 & 0 & 0 & 0 & 1 & 1 \\
\hline tweepy & 0 & 0 & 0 & 0 & 1 & 1 \\
\hline boto & 1 & 0 & 0 & 0 & 0 & 0 \\
\hline praw & 1 & 0 & 0 & 0 & 0 & 0 \\
\hline bs4 & 1 & 2 & 0 & 0 & 0 & 0 \\
\hline Total(Ratio) & $23(50 \%)$ & $3(6.52 \%)$ & $20(43.48 \%)$ \\
\hline
\end{tabular}

approaches do not. Our handling of the Python (dynamic) features, such as typing and attribute sets, is also novel.

Several tools have been developed for Python in bug detection $[2,3]$ and symbolic (concolic) execution [15, 17]. Bug detection tools are mainly based on static analysis and handle defects that do not involve dynamic features. Recent advances in Python symbolic (concolic) execution [15, 17] have shown good potentials. These techniques gradually explore paths within the search space, driven by a path exploration engine. In contrast, we use test cases to drive execution to interesting states and explore bugs in the neighborhood. Our state exploration is done inside the solver, not by a path exploration engine. As such, we need to address some new challenges such as encoding a variable in various types, handling unexecuted paths, and modeling attribute set changes. Type inference for Python [23, 24, 25] has also been proposed. The work is mainly based on abstract interpretation, which may have difficulty handling dynamic features.

A set of analyses have been proposed for other dynamic languages such as Javascript $[11,12,18,20]$, Ruby [14, 13, 19] and PHP [26, 21, 22]. TypeDevil [11] detects type inconsistencies through checking if a variable or field may have multiple types in the same run. Jalangi [18] supports concolic testing for Javascript which enables detecting type inconsistencies. Rubydust [13] dynamically infers types to report errors at method boundaries. Zheng et al. [22] proposed a path-sensitive static analysis of PHP web applications for security vulnerability detection.

\section{CONCLUSION}

In this paper, we present a Python predictive analysis. It has two steps to detect bugs in Python programs. The first step is to collect the execution trace of a passing run. The second is to encode the collected trace and some unexecuted branches into symbolic constraints. By solving the constraints, we can identify bugs as well as their triggering inputs. Our evaluation shows that our technique is able to detect 46 bugs with 16 unreported before. All of them are true positives.

\section{ACKNOWLEDGMENTS}

This research was partially supported by DARPA under contract FA8650-15-C-7562, NSF under awards 1409668, 1320444, and 1320306, ONR under contract N000141410468, Cisco Systems under an unrestricted gift, China Scholarship Council (CSC) Scholarship, National Basic Research Program of China (973 Program) No.2014CB340702, National Natural Science Foundation of China No.91418202, No.61472178 and No.91318301. Any opinions, findings, and conclusions in this paper are those of the authors only and do not necessarily reflect the views of our sponsors. 


\section{ARTIFACT DESCRIPTION}

We prepare our artifact package on a VMWare image which can be launched on a host with VMWare Workstation (version 10) installed. In this section, we provide detailed instructions about how to use the system.

Installation of VMWare Workstation. Our artifact image requires VMWare Workstation 10, which can be downloaded from [28]. Note that the Linux version of VMWare Workstation is packaged in a "*.bundle" executable so that one can simply use the command "sudo sh /path/to/<filename>. bundle" to start installation.

Download and Load the VM Image. First of all, go to the project website https://sites.google.com/site/ pypredictor/ to download the VM image. After decompression, please start VMware Workstation and go to menu File>0pen>Select the $\star . v m x$ file to load the image.

Artifact Contents in the Image. After loading the image, the HOME directory contains the following contents:

- A README file on the desktop (i.e., \$HOME/Desktop), which includes the requirements, instructions, evaluation and so on.

- The virtual environment (\$HOME/Pythons/virtualenvs) of Python with our tool and all the benchmarks installed.

- The working directory (\$HOME/Desktop/testcases), including all the test cases, configurations and running scripts.

- A mapping file issue-id-mapping.txt on the desktop, describing the mapping between test cases and the corresponding issue identifiers.

Source Code and Case Studies. We make the source code of our tool publicly available. It can be downloaded from the project website https://sites.google.com/ site/pypredictor/. Our tool can be installed on Python 2.7 (Linux). We also provide tutorials about how to install and run the system on a small buggy program. We have also put a number of case studies on the project website https://sites.google.com/site/pypredictor/ where we describe in details how these bugs occur, how our technique detects them and the analysis results of our tool.

Run the Tool. Before running the tool, please make sure the network of the VM works properly. Otherwise, the user may not get the right results of some cases. To start the tool, please use the following steps:

1. Open a terminal.

2. Switch to the Python virtual environment by: source \$HOME/Pythons/virtualenvs/python2.7.1/bin/activate.

3. Move to the working directory by command: cd \$HOME/Desktop/testcases.

4. Configure the Salt project by: ./saltconf.sh. Test the configuration by: salt $" \star "$ test.ping. If the test prints "True", the configuration was successful.

5. For a quick and full evaluation, use the command: ./run.sh. It will take 30-40 minutes to complete.

6. To evaluate the cases one by one, use the command: python test_caseX.py where $X$ means the ID of the test case.

We also provide the above instructions in the README file.
Evaluate the results. The evaluation goal is to reproduce the results shown in Table 3, which represents our main results. We have three kinds of output files during the execution of our tool. All of them are located in folder testcases/output by default. They are listed as follows:

- TraceMatrix_test_caseX.txt stores the detailed statistics of tracing results for test case $X$. Refer to README for the meaning of each record.

- PredictMatrix_test_casex.txt stores the detailed results of the predictive analysis. Also refer to README for the meaning of each record.

- PyTracer_test_caseX.db stores the dumped trace of test case $X$.

Refer to the issue mapping file issue-id-mapping.txt to align the results with those in the evaluation section. For more details about the evaluation, please refer to Section [Evaluation] in the README file.

\section{REFERENCES}

[1] IEEE Spectrum. http://spectrum.ieee.org/computing/ software/the-2015-top-ten-programming-languages.

[2] PyChecker. http://sourceforge.net/projects/pychecker/.

[3] PyLint. http://www.pylint.org/.

[4] Y. Smaragdakis, J. Evans, C. Sadowski, J. Yi, and C Flanagan. Sound predictive race detection in polynomial time. In 39th ACM SIGPLAN-SIGACT Symposium on Principles of Programming Languages (POPL), 2012.

[5] J. Huang, P. O. Meredith, and G. Rosu. Maximal sound predictive race detection with control flow abstraction. In 35th Annual Conference on Programming Language Design and Implementation (PLDI), 2014.

[6] M. Said, C. Wang, Z. Yang, and K. Sakallah. Generating data race witnesses by an smt-based analysis. In Third NASA Formal Methods Symposium (NFM), 2011.

[7] C. Wang, S. Kundu, M. Ganai, and A. Gupta. Symbolic predictive analysis for concurrent programs. In Formal Methods (FM), 2009.

[8] F. Sorrentino, A. Farzan, and P. Madhusudan. PENELOPE: weaving threads to expose atomicity violations. In 18th ACM SIGSOFT Symposium on the Foundations of Software Engineering (FSE), 2010.

[9] A. Farzan, P. Madhusudan, N. Razavi, and F. Sorrentino. Predicting null-pointer dereferences in concurrent programs. In 20th ACM SIGSOFT Symposium on the Foundations of Software Engineering (FSE), 2012.

[10] L. O. Andersen. Program Analysis and Specialization for the C Programming Language. PhD's thesis, $U C P H, 1994$.

[11] M. Pradel, P. Schuh, and K. Sen. TypeDevil: Dynamic type inconsistency analysis for JavaScript. In 37th International Conference on Software Engineering (ICSE), 2015.

[12] L. Gong, M. Pradel, M. Sridharan, and K. Sen. DLint: dynamically checking bad coding practices in JavaScript. In International Symposium on Software Testing and Analysis (ISSTA), 2015. 
[13] J. D. An, A. Chaudhuri, J. S. Foster, and M. Hicks. Dynamic inference of static types for Ruby. In 38th ACM SIGACT-SIGPLAN Symposium on Principles of Programming Languages (POPL), 2011.

[14] J. D. An, A. Chaudhuri, and J. S. Foster. Static Typing for Ruby on Rails. In 24th IEEE/ACM International Conference on Automated Software Engineering (ASE), 2009.

[15] T. Ball and J. Daniel Deconstructing Dynamic Symbolic Execution In Proceedings of the 2014 Marktoberdorf Summer School on Dependable Software Systems Engineering, 2014

[16] DART: directed automated random testing In $A C M$ SIGPLAN 2005 Conference on Programming Language Design and Implementation (PLDI), 2005.

[17] S. Bucur, J. Kinder, and G. Candea. Prototyping Symbolic Execution Engines for Interpreted Languages. In 19th International Conference on Architecture Support for Programming Languages and Operating Systems (ASPLOS), 2014.

[18] K. Sen, S. Kalasapur, T. Brutch, et al. Jalangi: A selective record-replay and dynamic analysis framework for JavaScript In 9th Joint Meeting of the European Software Engineering Conference and the ACM SIGSOFT Symposium on the Foundations of Software Engineering (ESEC/FSE), 2013.

[19] A. Chaudhuri and J.S. Foster. Symbolic security analysis of ruby-on-rails web applications. In 20th ACM Conference on Computer and Communications Security (CCS), 2010.

[20] S. Artzi, J. Dolby, S. H. Jensen, A. Møller, and F. Tip. A framework for automated testing of javascript web applications. In 33rd International Conference on Software Engineering (ICSE), 2011.

[21] E. Kneuss, P. Suter, and V. Kuncak. Runtime instrumentation for precise flow-sensitive type analysis. In 2nd International Conference on Runtime Verification (RV), 2011.

[22] Y Zheng, X. Zhang. Path sensitive static analysis of web applications for remote code execution vulnerability detection In 35th International Conference on Software Engineering (ICSE), 2013.

[23] M .Salib. Starkiller: A static type inferencer and compiler for python. In Master's thesis, MIT, 2004.

[24] A. Rigo and S. Pedroni. Pypy's approach to virtual machine construction. In ACM SIGPLAN International Conference on Object Oriented Programming Systems Languages \& Applications (OOPSLA), 2006.

[25] M. Gorbovitski, Y. A. Liu, S. D. Stoller, T. Rothamel, and K. T. Tekle. Alias analysis for optimization of dynamic languages. In Dynamic Languages Symposium (DLS), 2010.

[26] H. Zhao, I. Proctor, M. Yang, X. Qi, M. Williams, et al. The hiphop compiler for php. In ACM SIGPLAN International Conference on Object Oriented Programming Systems Languages \& Applications (OOPSLA), 2012.

[27] Python Predictive Analysis https://sites.google.com/site/pypredictor/.

[28] VMWare Workstation https: //my.vmware.com/web/vmware/info?slug=desktop_ end_user_computing/vmware_workstation/10_0. 